\title{
Efficacy and safety of a three-times-weekly dosing regimen of glatiramer acetate in relapsing-remitting multiple sclerosis patients: 3-year results of the Glatiramer Acetate Low-Frequency Administration open-label extension study
}

Omar Khan, Peter Rieckmann, Alexey Boyko, Krzysztof Selmaj, Natalia Ashtamker, Mat D Davis, Scott Kolodny and Robert Zivadinov

\section{Abstract}

Background: The 1-year placebo-controlled (PC) phase of the Glatiramer Acetate Low-Frequency Administration (GALA) study showed that glatiramer acetate $40 \mathrm{mg} / \mathrm{mL}$ three times weekly (GA40) significantly reduced annualized relapse rate (ARR) and magnetic resonance imaging (MRI) activity in patients with relapsing-remitting multiple sclerosis. Patients completing the PC phase were invited to an open-label (OL) extension.

Objective: To evaluate the effects of early start (ES) and delayed start (DS) of GA40 over 3 years.

Methods: A total of $97.2 \%$ of patients completing the PC phase received GA40 in the OL extension. ES $(n=943)$ patients received GA40 throughout; DS $(n=461)$ patients received placebo during the PC phase and GA40 during the OL phase. Relapse, MRI, disease progression, and safety were evaluated.

Results: A total of 1041 patients completed 3 years of follow-up. During the OL phase, ES and DS patients showed comparable ARRs (0.20-0.22) and similar numbers of gadolinium-enhancing T1 $(p=0.49)$ and new or enlarging T2 lesions ( $p=0.51)$ at Year 3. ES patients showed significantly smaller changes in gray matter volume than DS patients from Months 12 to 36 (mean difference, $0.371 \% ; p=0.015$ ), with similar trend in whole-brain volume $(p=0.080)$. Adverse events were mild, consistent with the well-established glatiramer acetate (GA) safety profile.

Conclusion: GA40 conferred treatment benefit over 3 years: sustained low ARR and lesion activity and favorable safety.

Keywords: Glatiramer acetate, relapsing-remitting multiple sclerosis, annualized relapse rate, lowfrequency regimen, safety

Date received: 10 February 2016; revised: 13 June 2016; accepted: 30 June 2016

Introduction

Glatiramer acetate (GA) is a first-line therapy approved for the treatment of relapsing-remitting multiple sclerosis (RRMS) that has a well-characterized long-term safety profile and established efficacy, with more than 2 million patient-years of overall exposure to glatiramer acetate $20 \mathrm{mg} / \mathrm{mL}$ administered daily by subcutaneous injection (GA20). ${ }^{1-4}$ Like other first-line treatment regimens in RRMS, GA20 requires longterm injection of the drug and can be associated with injection-related adverse events (IRAEs), which may diminish adherence in some patients. ${ }^{5-7}$ This is of particular importance because treatment adherence ensures optimal clinical outcomes. ${ }^{8}$ Modified treatment regimens with proven, long-term clinical efficacy have the potential to minimize adverse side
Multiple Sclerosis Journal 2017, Vol. 23(6) 818-829 DOI: 10.1177 1352458516664033

(c) The Author(s), 2016. Reprints and permissions: http://www.sagepub.co.uk/ journalsPermissions.nav
Correspondence to: S Kolodny

Sr Medical Dir, Global Medical Affairs, Teva Pharmaceuticals, 401 Moores Rd, Frazer, PA 19355, USA. scott.kolodny@tevapharm. com

Omar Khan The Sastry Foundation Advanced Imaging Laboratory \& Multiple Sclerosis Center, Department of Neurology, Wayne State University School of Medicine, Detroit, MI, USA Peter Rieckmann Department of Neurology, Bamberg Academic Hospital, University of Erlangen, Bamberg, Germany Alexey Boyko Department of Neurology, Neurosurgery and Medical Genetic of the Pirogov's Russian National Research Medical University and MS Clinic at the Usupov's Hospital, Moscow, Russia

Krzysztof Selmaj Department of Neurology, Medical University of Łódź, Łódź, Poland

Natalia Ashtamker Teva Pharmaceutical Industries, Netanya, Israel Mat D Davis Teva Pharmaceuticals, Frazer, PA, USA Scott Kolodny Teva Pharmaceuticals, Cleveland, OH, USA

Robert Zivadinov Department of Neurology, School of Medicine and Biomedical Sciences, State University of New York at Buffalo, Buffalo, NY, USA 
effects while maintaining efficacy, thereby supporting appropriate adherence to treatment and eliciting greater overall clinical benefit. ${ }^{1,9,10}$

The Glatiramer Acetate Low-Frequency Administration (GALA) study was a Phase III trial conducted to investigate the efficacy and safety of a subcutaneous injection of glatiramer acetate $40 \mathrm{mg} / \mathrm{mL}$ administered three times weekly (GA40) to patients with RRMS. ${ }^{11}$ In 2014, based on the placebo-controlled (PC) phase results of the GALA study, the GA40 regimen was approved for the treatment of RRMS. The GA40 regimen had efficacy and safety profiles similar to those of the established daily GA20 regimen. ${ }^{11}$

The PC phase results of the GALA study showed that GA40 significantly reduced the annualized relapse rate (ARR) and the number of cumulative gadolinium-enhancing (GdE) T1 and new or enlarging T2 lesions in patients with RRMS over 1 year compared with placebo. ${ }^{11}$

Participants who completed the 1-year PC phase of the GALA study were eligible to receive GA40 treatment in an ongoing open-label (OL) extension study and were invited to switch to or continue the GA40 regimen. This clinical assessment of GA40 will provide further insight into the value of the three-timesweekly dosing regimen in the treatment of RRMS.

\section{Materials and methods}

\section{Study design and patients}

GALA was a randomized, PC, double-blind, parallelgroup study conducted at 142 sites in 17 countries. All institutional review boards or ethical committees of the participating centers approved the protocol, and all patients gave written informed consent before any study-related procedures were performed. Study design, eligibility criteria, and conduct of the PC phase of the GALA study have been reported previously. ${ }^{11,12}$ During the PC phase, eligible patients were randomized 2:1 to receive GA40 or placebo and seven scheduled site visits occurred: at screening, baseline (Month 0), and Months 1, 3, 6, 9, and 12.

Study participants from either treatment group were eligible for participation in the OL phase if they completed the PC phase according to the protocol, and all patients participating in the OL phase were treated with GA40 until it became commercially available for the treatment of RRMS or development was stopped by the sponsor. Patients who received GA40 for the duration of the study were referred to as early start
(ES) patients, and those who received placebo during the PC phase and had the option to convert to GA40 at the start of the OL phase were referred to as delayed start (DS) patients.

During the OL extension phase, site visits were scheduled to occur every 3 months for the first year and every 6 months thereafter. Procedures performed at each scheduled visit during the OL extension included assessment of vital signs and evaluation of relapse. A complete neurological and physical examination was performed every 6 months, and electrocardiograms (ECGs), safety laboratory tests, and serum pregnancy tests were performed annually. Magnetic resonance imaging (MRI) scans were performed at baseline, Month 6, Month 12, and Month 36 of the study. Adverse events (AEs) and the use of concomitant medications were monitored throughout the study.

\section{Clinical endpoints}

The primary endpoint of the PC phase was the ARR, defined as the total number of confirmed relapses divided by the annual exposure to study drug. Patients with symptoms suggestive of a relapse were instructed to contact the study site within 48 hours of symptom onset and underwent a complete neurological assessment, performed by the examining neurologist or physician. A relapse was defined as the appearance of one or more new neurological abnormalities, or the reappearance of one or more previously observed neurological abnormalities, lasting at least 48 hours and immediately preceded by an improvement in neurological state lasting at least 30 days from the onset of the previous relapse. An event was counted as a relapse only when the subject's symptoms were accompanied by observed objective neurological changes, including an increase of $\geqslant 0.5$ in the Expanded Disability Status Scale (EDSS) score compared with the previous evaluation, an increase of one grade in the score of $\geqslant 2$ of the seven functional systems (FSs) compared with the previous evaluation or an increase of two grades in the actual score of one FS compared with the previous evaluation. The patient must not have had any acute metabolic changes, such as fever or other medical abnormality, and a change in bowel or bladder function or cognitive function must not have been entirely responsible for confirmation of a relapse. On confirmation of relapse, follow-up of patients' neurological condition was performed with scheduled and unscheduled visits.

Additional clinical endpoints included time to first relapse, time to 6-month confirmed disability progression (CDP), and time to 6-month confirmed 
EDSS 4. Six-month confirmed progression was defined as an increase in EDSS score of $\geqslant 1$ point from placebo-controlled baseline (PCBL) if EDSS score at PCBL was $\leqslant 5.0$, confirmed after at least 6 months, or an increase of $\geqslant 0.5$ points from PCBL if EDSS score at PCBL was $\geqslant 5.5$, confirmed after at least 6 months. Progression could not be confirmed during a relapse. Time to 6-month confirmed EDSS 4 was defined as an increase in EDSS score to $\geqslant 4$ for subjects with baseline EDSS score $<4$ confirmed after at least 6 months.

MRI endpoints included number of GdE T1 lesions, number of new or enlarging T2 lesions, percent brain volume change (PBVC) from baseline to Month 36 and from Months 12 to 36, and percent change in gray matter (GM) and white matter (WM) volumes from baseline to Month 36 and from Months 12 to 36. Endpoints also included percent volume changes of the total deep GM and of the thalamus at each time point.

\section{Safety endpoints}

Safety was assessed by the incidence of AEs, serious AEs, changes in vital signs, clinical laboratory parameters, and ECG findings over time. An immediate post-injection reaction (IPIR) was defined as an adverse reaction consisting of one or more of the following symptoms: vasodilatation, chest pain, dyspnea, palpitations, or tachycardia.

\section{MRI methodology}

Before study participants were scanned, MRI facilities underwent a qualification procedure to ensure that appropriate images were produced for measuring the endpoints specified by the study protocol. The following MRI scans were obtained according to a standard protocol provided by the MRI reading center (Buffalo Neuroimaging Analysis Center, Buffalo, NY): two-dimensional (2D) T2-weighted image (WI), 2D proton density-weighted image (PDWI), three-dimensional (3D) T1-WI, fluid attenuated inversion recovery (FLAIR), and spin-echo T1-WI with and without Gd contrast. All MRI scans were transferred to the MRI reading center, where they were quantitatively evaluated. All images were $3 \mathrm{~mm}$ thick, except the 3D T1-WI, which was $1.5 \mathrm{~mm}$ thick. To the greatest extent possible, additional geometry and other sequence parameters were standardized between the sites. All MRI scans were interpreted in a blinded manner.

T2 FLAIR and GdE T1 lesions were assessed. ${ }^{13}$ All within-subject images were co-registered to baseline using FMRIB's Linear Image Registration Tool (FLIRT). ${ }^{14}$ All subsequent lesion analyses were done using the co-registered images. Trained expert raters primarily used the FLAIR and T1 post-contrast images, along with supporting images including PD, $\mathrm{T} 2$, and longitudinal subtraction images (produced via voxel-wise subtraction of the prior time point). The volume of $\mathrm{T} 2$ lesions at baseline was calculated using a semi-automated contouring or thresholding technique. ${ }^{15}$

For volumetric analyses, 3D-T1 images were preprocessed using a lesion-inpainting algorithm, to minimize the impact of WM lesions on tissue volume measurements. All MRI analyses underwent multilevel quality control, and MRI scans were reviewed by trained operators at all critical points and were either corrected, if possible, or excluded from further analysis. At baseline, normalized whole-brain, GM (composed of cortical GM and deep GM nuclei), and WM volumes were measured using the SIENAX technique. ${ }^{16}$ The PBVC (whole brain) between different time points was assessed using the structural image evaluation normalization of atrophy (SIENA) method, ${ }^{17}$ and percentage changes in GM (composed of cortical GM and deep GM nuclei) and WM volumes were calculated using the SX-MTP (SIENAX multi-time point) method. ${ }^{18}$ The baseline assessment and estimation of percent volume changes between each time point of the total deep GM (defined as the sum of thalamus, caudate nucleus, putamen, globus pallidus, hippocampus, amygdala, and nucleus accumbens) and of the thalamus nuclei were performed using FMRIB's Integrated Registration and Segmentation Tool (FIRST). ${ }^{19,20}$

\section{Statistical analysis}

The analysis of efficacy outcomes was performed in the intention-to-treat analysis set, defined as all randomized patients. Analysis of safety outcomes was performed in the safety analysis set, defined as all subjects who received at least one dose of GA40 in either the PC or OL phase (Figure 1).

The analysis of the primary endpoint, ARR, was based on estimating a contrast (ES vs DS) derived from a repeated-measures, baseline-adjusted negative binomial regression model of the number of confirmed relapses per year from baseline through the $\mathrm{OL}$ extension phase. In addition to treatment group and year on study, the negative binomial regression model included the following covariates as predictors: baseline EDSS score, log of the number of relapses in the previous 2 years, volume of $\mathrm{T} 2$ lesions at baseline, 


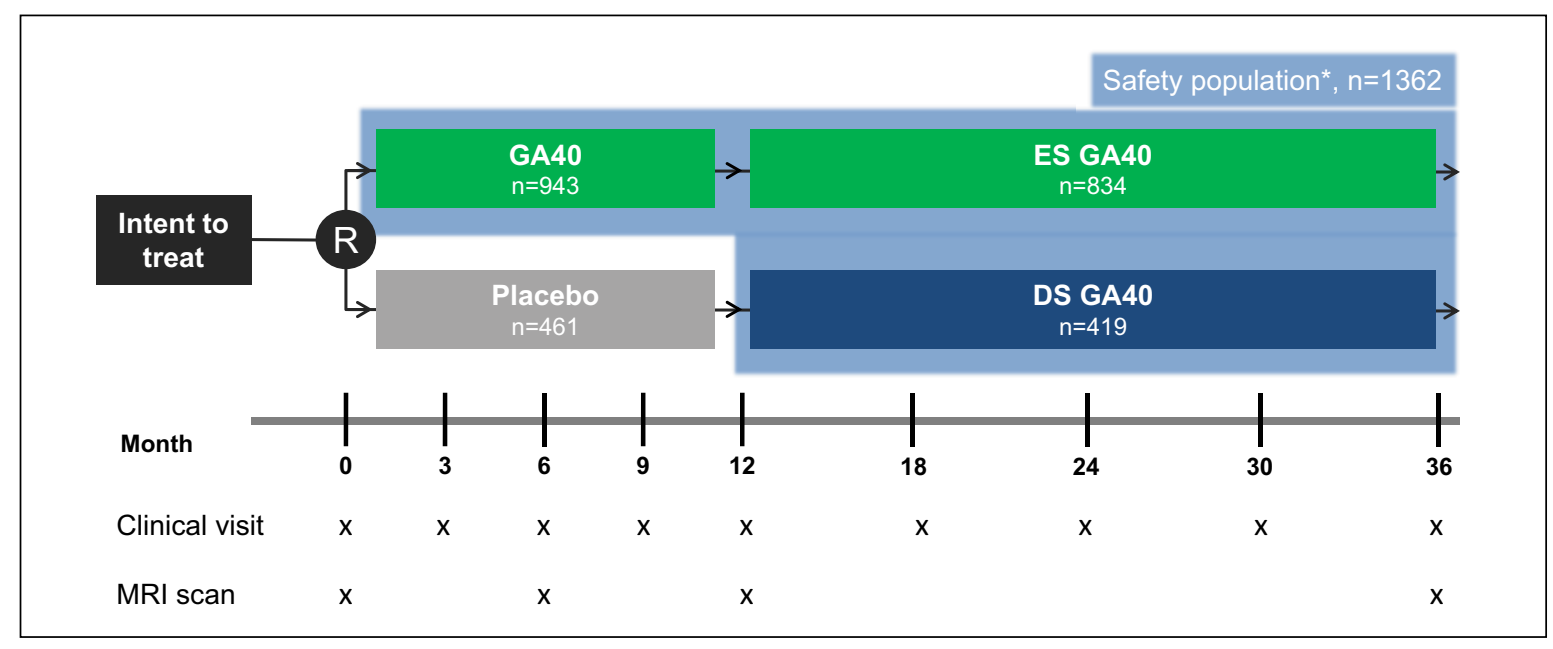

Figure 1. Study design. Only clinical visits involving complete neurological and physical examination are represented here.

DS: delayed start; ES: early start; GA40: glatiramer acetate $40 \mathrm{mg} / \mathrm{mL}$ subcutaneous injection administered three times weekly; OL: open-label; PC: placebo-controlled.

*Patients exposed to GA40 (received at least one dose) at any point during the study.

status of GdE T1 activity at baseline, country or geographical region, and treatment by year interaction.

Time to first relapse was analyzed using a Cox proportional hazards model and summarized by a hazard ratio (HR) derived from the outcome of a contrast (ES vs DS); the model was adjusted for baseline EDSS score, log of the number of relapses in the previous 2 years, volume of T2 lesions at baseline, status of GdE T1 activity at baseline, and country or geographical region. Time to 6-month CDP and 6-month confirmed EDSS 4 was analyzed using the outcome of a contrast (ES vs DS) derived from a baseline-adjusted Cox proportional hazard model; in addition to treatment group, the model was adjusted for baseline EDSS score and country or geographical region.

Analysis of MRI endpoints, the number of GdE T1 lesions, and the number of new or enlarging T2 lesions, at each visit for each treatment group, used a repeated-measures negative binomial regression model with the number of lesions at each scan as the outcome and the baseline number of GdE T1 lesions, country or geographic region, and treatment by scan interaction as predictors. The analyses of percent change in whole-brain, GM, or WM volumes were based on the outcome of a contrast (ES vs DS) derived from a baseline-adjusted analysis of covariance. In addition to treatment group, the model also included SIENAX-normalized whole-brain volume at baseline, number of enhancing lesions on T1-WIs at baseline, and country or geographical region as covariates. Safety and tolerability results were reported descriptively.

\section{Results}

\section{Patients}

The vast majority $(97.2 \%, 1253$ of 1289$)$ of patients who completed the double-blind, 1-year PC phase consented to receive GA40 in the OL extension (Figure 2). ES patients $(n=943)$ received GA40 throughout, whereas DS patients $(n=461)$ were randomized to placebo and switched to GA if they entered the OL phase (Figure 2). A total of $716(75.9 \%)$ ES patients and 325 (70.5\%) DS patients completed 3 years of follow-up, and $562(59.6 \%)$ ES patients and $260(56.4 \%)$ DS patients completed the Year 3 MRI scan (Figure 2). Most discontinuations in the OL phase were due to withdrawal of consent (143 (11\%) in all OL patients: $84(10 \%)$ in ES and 59 (14\%) in DS group). Of these patients, 141 offered additional insight into the reason for withdrawal of consent with "patient decision" (53 of 141 responses) and "lack of efficacy" (32 of 141 responses) recorded as the two most common reasons for withdrawal. A total of 1362 patients were exposed to GA40 (received at least one dose) at any point during the study (Figure 1). Baseline demographics and disease characteristics showed no significant differences between treatment groups (Table 1). 


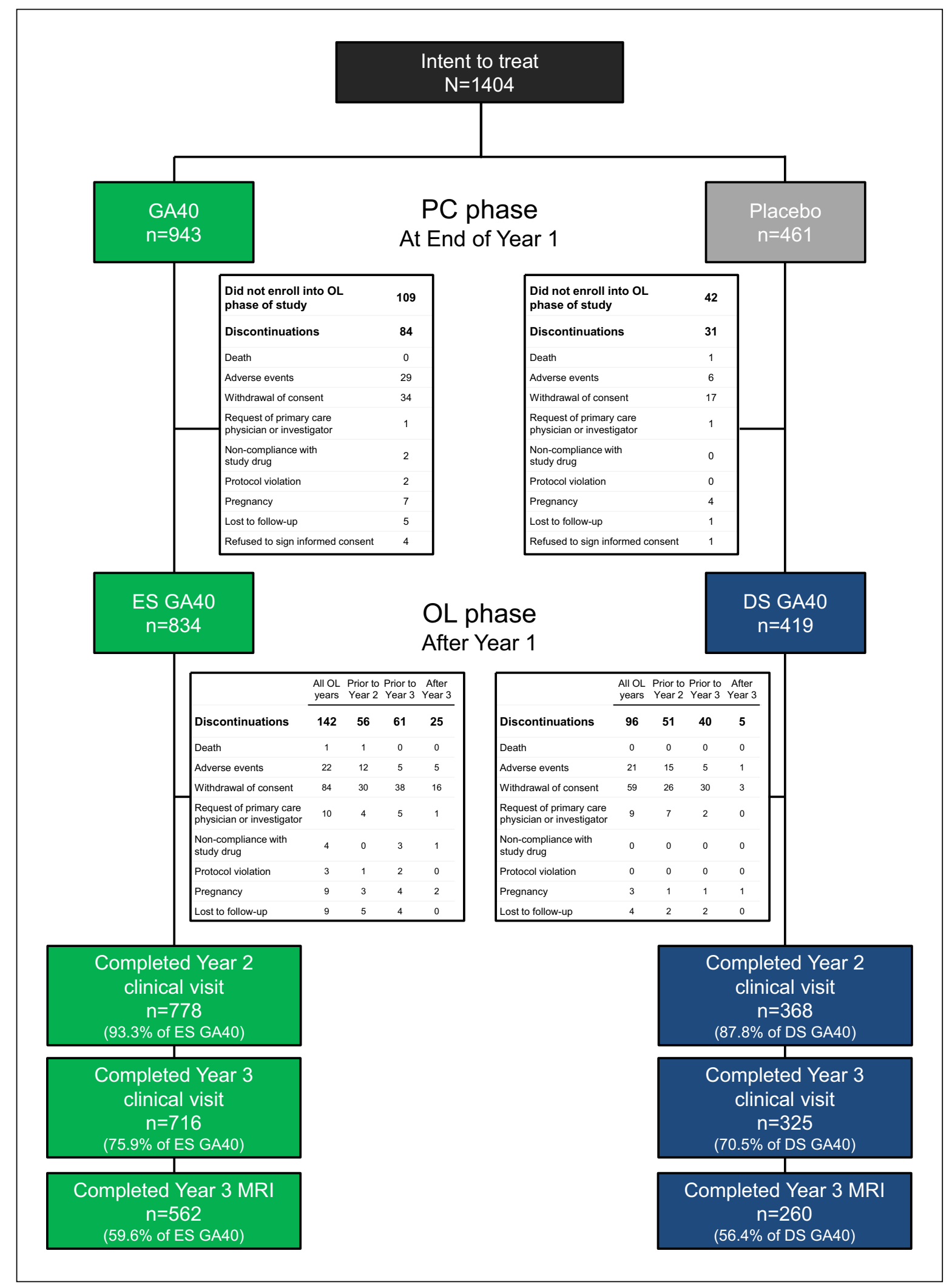

Figure 2. Patient disposition.

DS: delayed start; ES: early start; GA40: glatiramer acetate $40 \mathrm{mg} / \mathrm{mL}$ subcutaneous injection administered three times weekly; OL: open-label; PC: placebo-controlled. 
Table 1. Baseline patient and disease characteristics. ${ }^{\mathrm{a}}$

\begin{tabular}{lll}
\hline & $\begin{array}{l}\text { GA40/ES patients } \\
(n=943)\end{array}$ & $\begin{array}{l}\text { Placebo/DS patients } \\
(n=461)\end{array}$ \\
\hline Age, years, mean \pm SD & $37.4 \pm 9.4$ & $38.1 \pm 9.2$ \\
Female gender, $n(\%)$ & $641(68.0)$ & $313(67.9)$ \\
EDSS, mean \pm SD & $2.8 \pm 1.2$ & $2.7 \pm 1.2$ \\
Caucasian, $n$ (\%) & $916(97.1)$ & $455(98.7)$ \\
Body mass index, mean \pm SD & $24.4 \pm 4.7$ & $24.4 \pm 4.8$ \\
Years from onset of first MS symptoms, mean \pm SD & $7.7 \pm 6.7$ & $7.6 \pm 6.4$ \\
Exacerbations over 2 years prior to study initiation, mean \pm SD & $1.9 \pm 0.9$ & $1.9 \pm 0.9$ \\
Number of GdE T1 lesions, mean \pm SD & $1.7 \pm 4.7$ & $1.4 \pm 3.7$ \\
Patients with $>0$ GdE T1 lesions, $n(\%)$ & $336(35.6)$ & $154(33.4)$ \\
Volume (cc) of T2 lesion, mean \pm SD & $19.7 \pm 20.7$ & $17.4 \pm 17.4$ \\
Normalized whole-brain volume (cc), mean \pm SD & $1533.9 \pm 110.6$ & $1537.9 \pm 110.8$ \\
Normalized GM volume (cc), mean \pm SD & $801.9 \pm 68.3$ & $803.8 \pm 68.9$ \\
Normalized WM volume (cc), mean \pm SD & $731.3 \pm 64.4$ & $733.9 \pm 64.5$ \\
\hline DS: delayed start; EDSS: Expanded Disability Status Scale; ES: early start; GA40: glatiramer acetate $40 \mathrm{mg} / \mathrm{mL}$ subcutaneous injec- \\
tion administered three times weekly; GdE: gadolinium-enhancing; GM: gray matter; ITT: intent to treat; SD: standard deviation; \\
WM: white matter; MS: multiple sclerosis. \\
aNo significant differences between the two groups at baseline.
\end{tabular}

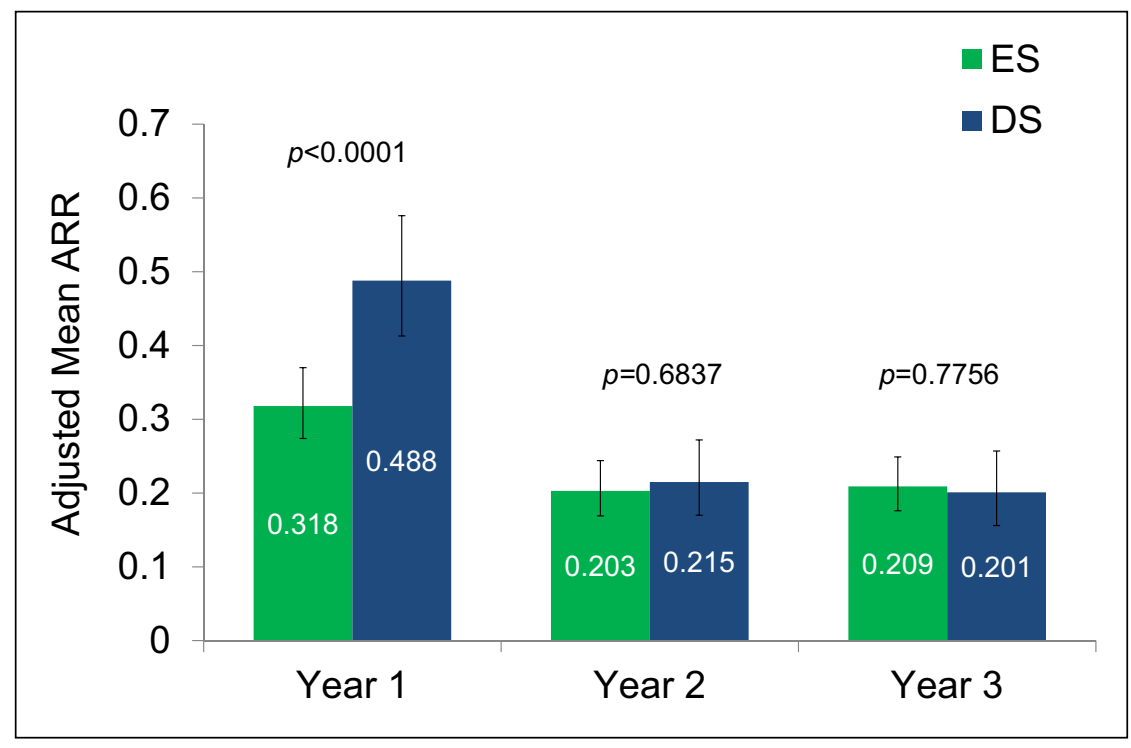

Figure 3. Annualized relapse rate. Error bars represent $95 \%$ confidence interval. ARR: annualized relapse rate; DS: delayed start; ES: early start.

ARR

The mean ARR was significantly lower for ES patients than for DS patients over the first year of treatment (Year 1 ; risk ratio $(\mathrm{RR})=0.652 ; 95 \%$ confidence interval $(\mathrm{CI})$ : $0.537-0.793 ; p<0.0001$; Figure 3$)$. During the OL extension phase, following the conversion from placebo to GA40 in the DS group, ARR was similar between ES patients and DS patients at Year $2(\mathrm{RR}=0.944$; 95\% CI:
$0.716-1.245 ; p=0.68)$ and at Year $3(\mathrm{RR}=1.043 ; 95 \%$ CI: $0.782-1.391 ; p=0.78$; Figure 3 ).

\section{Time to first relapse}

Time to first relapse was significantly longer in ES patients compared with DS patients $(\mathrm{HR}=0.746 ; 95 \%$ CI: $0.628-0.887 ; p=0.0009$; Figure 4). 


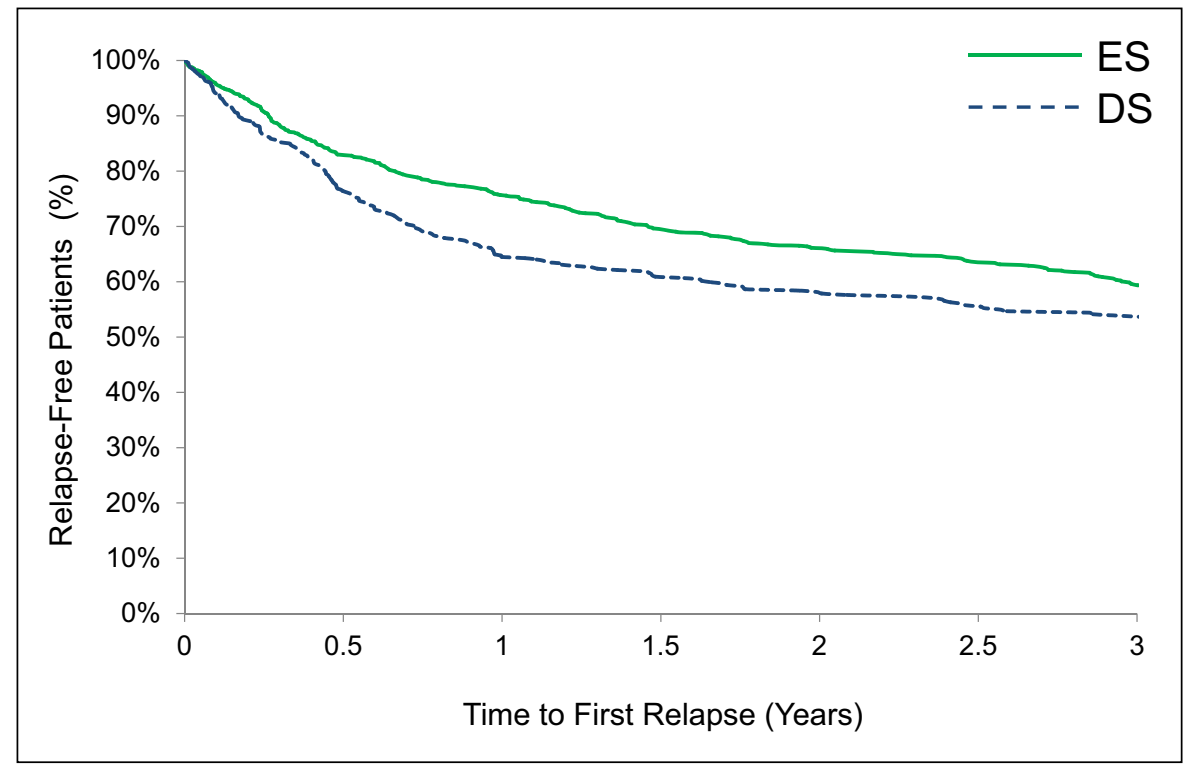

Figure 4. Time to first relapse.

DS: delayed start; ES: early start.

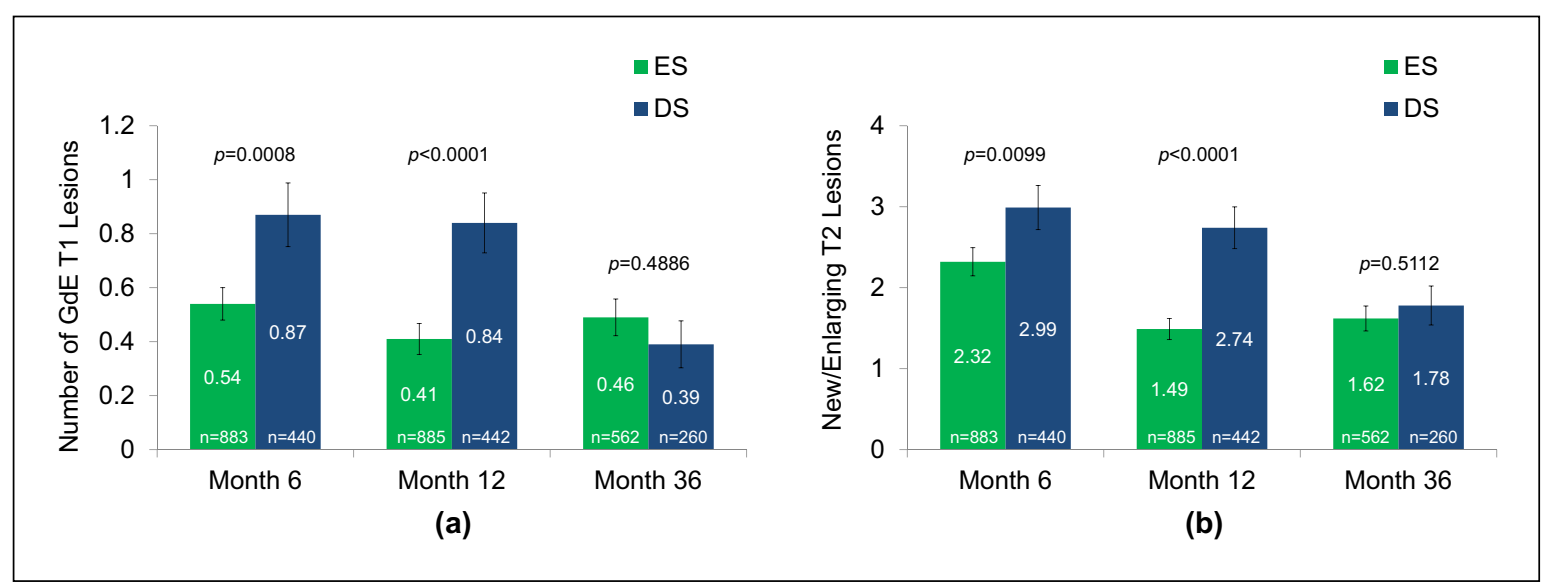

Figure 5. (a) Adjusted mean number of GdE T1 lesions and (b) new or enlarging T2 lesions. Error bars represent standard error.

DS: delayed start; ES: early start; GdE: gadolinium-enhancing.

\section{Time to CDP}

Overall, there were low disability event rates, with $11 \%$ of ES patients and $13 \%$ of DS patients experiencing 6-month CDP during the entire study, including the $\mathrm{PC}$ and $\mathrm{OL}$ phases $(\mathrm{HR}=0.759 ; 95 \% \mathrm{CI}$ : $0.552-1.044 ; p=0.09)$. There were similarly low progression rates for 6-month confirmed EDSS 4 with $3 \%$ of ES patients and $5 \%$ of DS patients ( $\mathrm{HR}=0.559$; 95\% CI: $0.319-0.979 ; p=0.042$ ).

\section{Number of MRI lesions}

The number of GdE T1 lesions and new or enlarging T2 lesions was significantly lower for ES patients than for DS patients over the first year of treatment, when evaluated at Months 6 and 12 (Figure 5). During the OL extension phase, following the conversion from placebo to GA40 in the DS group, lesion counts were similar between ES patients and DS patients at Year 3 (GdE T1 lesions: RR=1.19; 95\% CI: 0.728 1.946; $p=0.49$; new or enlarging $\mathrm{T} 2$ lesions: $\mathrm{RR}=0.907 ; 95 \%$ CI: 0.677-1.214; $p=0.51$; Figure 5).

\section{Changes in brain volume}

At baseline, there were no differences between the ES and DS patients in normalized whole-brain, GM, and WM volumes (Table 1) and in deep GM volumes 


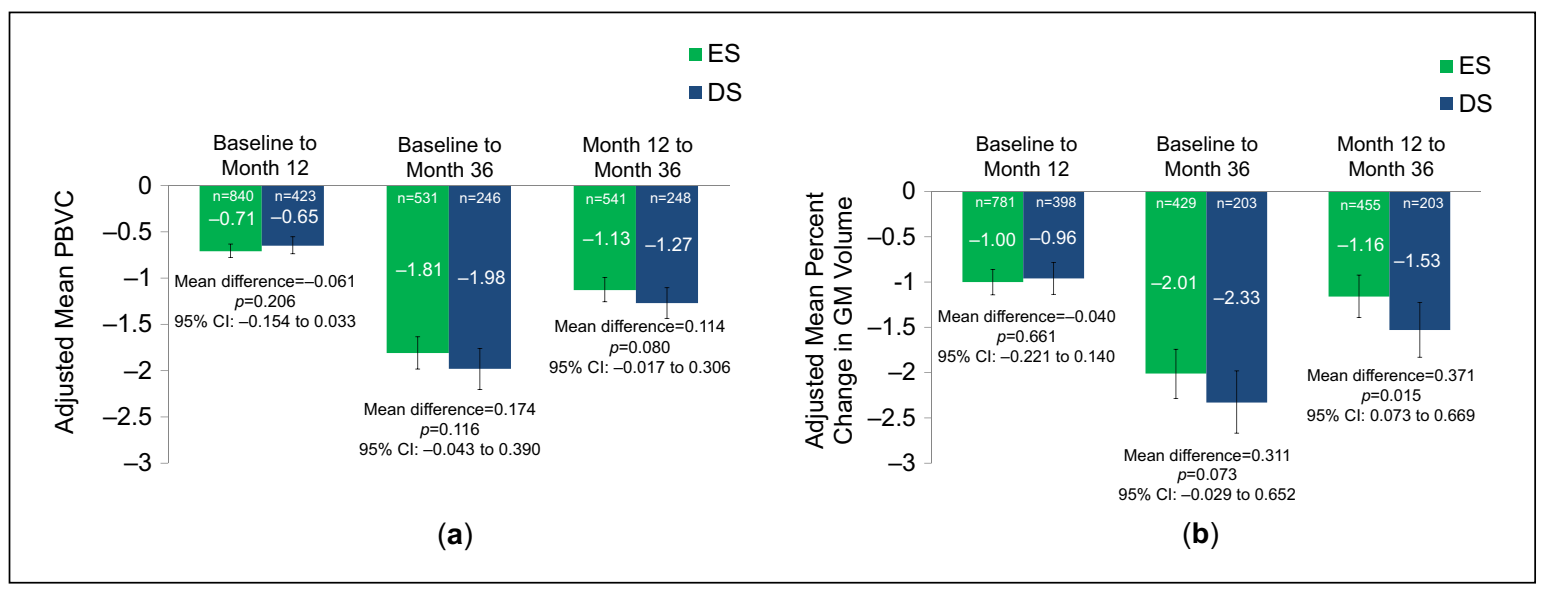

Figure 6. Changes in brain volume. Because both (a) PBVC (whole-brain) and (b) GM (composed of cortical GM and deep GM nuclei) volume change analyses were performed using direct methods of brain atrophy assessment (SIENA and SX-MTP), the pair of scans used between baseline to Month 36 and Months 12-36 were not identical. For both PBVC and GM volume change analyses, a greater portion of the Months 12-36 analyses passed final quality control than did the baseline to Month 36 analyses. Error bars represent standard error.

CI: confidence interval; DS: delayed start; ES: early start; GM: gray matter.

(data not shown). During the core phase, there were no statistical differences between ES and DS patients in adjusted mean PBVC (baseline to Month 12: $-0.706 \%$ vs $-0.645 \%$, respectively; $p=0.21$ ), adjusted mean WM volume change (baseline to Month 12: $-0.245 \%$ vs $-0.293 \%$, respectively; $p=0.59$ ), or adjusted mean GM volume change (baseline to Month 12 : $-1.001 \%$ vs $-0.961 \%$, respectively; $p=0.66$ ). In the OL phase, there was a trend toward a decreased loss of whole-brain volume in ES patients compared with DS patients (baseline to Month 36: $-1.81 \%$ vs $-1.98 \%$, respectively; $p=0.12$; Months $12-36$ : $-1.13 \%$ vs $-1.27 \%$, respectively; $p=0.08$; Figure 6(a)). At Month 36, ES patients showed less GM volume loss compared with DS patients (baseline to Month 36 : $-2.01 \%$ vs $-2.33 \%$, respectively; $p=0.07$; Months $12-36$ : $-1.16 \%$ vs $-1.53 \%$, respectively; $p=0.015$; Figure 6(b)). No significant differences were observed in WM, thalamic, or deep GM volume changes between groups (data not shown).

\section{Safety}

GA40 was well tolerated, with no new safety signals identified. AEs were consistent with the well-established GA safety profile. There were no relevant safety signals with regard to hematologic, biochemical, or urinalysis laboratory values, ECG readings, or vital signs. Over three-quarters of patients exposed to GA40 experienced at least one AE, whereas $8 \%$ experienced a serious $\mathrm{AE}$ (Table 2). The most frequently occurring systemic AEs included headache, back pain, and infections, such as nasopharyngitis.
Table 2. Frequency of common adverse events in all patients exposed to GA40. ${ }^{\text {a }}$

\begin{tabular}{|c|c|}
\hline & $\begin{array}{l}\text { Total GA40 }(N=1362 ; \\
\mathrm{PY}=3446.4)\end{array}$ \\
\hline $\begin{array}{l}\text { Total number of patients with } \\
\text { serious AEs, } n(\%)\end{array}$ & $111(8.1)$ \\
\hline $\begin{array}{l}\text { Total number of patients with } \\
\text { AEs, } n(\%)\end{array}$ & $1032(75.8)$ \\
\hline Influenza & $82(6.0)$ \\
\hline Nasopharyngitis & $197(14.5)$ \\
\hline $\begin{array}{l}\text { Upper respiratory tract } \\
\text { infections }\end{array}$ & $107(7.9)$ \\
\hline Urinary tract infection & $105(7.7)$ \\
\hline Back pain & $97(7.1)$ \\
\hline Headache & $151(11.1)$ \\
\hline $\begin{array}{l}\text { Total number of patients with } \\
\text { injection-site reactions, } n(\%)\end{array}$ & $542(39.8)$ \\
\hline Injection-site erythema & $351(25.8)$ \\
\hline Injection-site pain & $169(12.4)$ \\
\hline Injection-site pruritus & $93(6.8)$ \\
\hline Injection-site swelling & $75(5.5)$ \\
\hline Injection-site atrophy ${ }^{b}$ & $22(1.6)$ \\
\hline \multicolumn{2}{|c|}{$\begin{array}{l}\text { AE: adverse event; GA40: glatiramer acetate } 40 \mathrm{mg} / \mathrm{mL} \\
\text { subcutaneous injection administered three times weekly; PY: } \\
\text { patient-years. } \\
\text { aIndividual AEs reported in this table (preferred term) are } \\
\text { those occurring in more than } 5 \% \text { of patients exposed to GA40 } \\
\text { at any time during the study, with the exception of injection- } \\
\text { site atrophy. AEs reported here are those occurring from the } \\
\text { start of GA40 until the last dose in the study. } \\
\text { bInjection-site atrophy (categorical term) includes injection- } \\
\text { site atrophy, injection-site lipoatrophy, and injection-site } \\
\text { lipodystrophy acquired. }\end{array}$} \\
\hline
\end{tabular}


Table 3. Immediate post-injection reactions (IPIRs) in all patients exposed to GA40. ${ }^{\text {a }}$

\begin{tabular}{llll} 
& \multicolumn{2}{c}{ Total GA40 $(N=1362 ;$ PY=3446.4) } \\
\cline { 2 - 4 } & No. of patients, $n(\%)$ & No. of events & Incidence rate per 100 PY \\
\hline Total & $142(10.4)$ & 272 & 4.1 \\
Dyspnea & $56(4.1)$ & 79 & 1.6 \\
Vasodilation (flushing) & $47(3.5)$ & 75 & 1.4 \\
Tachycardia & $41(3.0)$ & 57 & 1.2 \\
Chest pain & $35(2.6)$ & 43 & 1.0 \\
Palpitations & $16(1.2)$ & 18 & 0.5 \\
\hline GA40: glatiramer acetate $40 \mathrm{mg} / \mathrm{mL}$ subcutaneous injection administered three times weekly; PY: patient-years. \\
aIPIRs reported here are those occurring at the start of GA40 until the last dose in the study. \\
\hline
\end{tabular}

The incidence of local injection-site reactions was $39.8 \%$, with injection-site atrophy occurring least frequently ( $1.6 \%$ incidence). At least one event related to systemic IPIRs occurred in $10.4 \%$ of all patients exposed to GA40 (number of events: 272; incidence rate: 4.1 per 100 patient-years). Dyspnea and vasodilation were the most common reported IPIRs (Table 3).

There was one death during the OL phase due to cardiac failure in a 41-year-old obese male smoker with a prior history of myocardial infarction. Postmortem evaluation showed that this patient had post-infarction cardiosclerosis, and death was considered not to be related to study drug by both the investigator and the study sponsor.

\section{Discussion}

The results of the 3-year OL extension phase of the GALA study support the benefit of GA40 sustained beyond that reported in the PC phase of the trial. ${ }^{11}$ Relapse-related efficacy seen in GALA for GA40 was generally similar to that seen in previous studies with GA20, in which reductions in ARR were $29 \%-$ $33 \% .{ }^{21,22}$ However, given the variation between study designs, caution should be exercised when comparing prior GA20 studies and GALA. During OL treatment, the GA40 efficacy effect experienced by the ES group during the PC phase was maintained. After initiation of GA40 in the OL phase, DS patients experienced a treatment benefit in ARR comparable to that of the ES group and consistent with the observed treatment effect of GA40 during the PC phase.

Overall, there were low rates of disability progression events; however, patients with early initiation of GA40 showed a trend toward a delayed progression to 6-month confirmed EDSS $4(\mathrm{HR}=0.559 ; 95 \% \mathrm{CI}$ : $0.319-0.979 ; p=0.042$ ). Progression to milestone EDSS scores, and maintenance of such changes over several months, may reflect an irreversible accumulation of disability. ${ }^{23}$ The trends observed in this 3-year study would need to be confirmed following additional exposure and observation of higher rates of disability progression.

The MRI outcomes of this study corroborate the clinical benefits of GA40. The small number of GdE T1 lesions and new or enlarging T2 lesions at Months 6 and 12 observed in patients treated with GA40 during the PC phase was sustained throughout the OL phase to Month 36. For the DS group, conversion to GA40 from placebo at the start of the OL phase resulted in a reduction in MRI lesion activity from what was observed during the PC phase. Sustained reduction of the number of MRI lesions and relapse activity is consistent with the known long-term experience with GA20, and importantly, no signals of increased disease activity were noted with GA40.

During the PC phase, there was no difference in PBVC or GM volume percentage change between ES and DS patients. This is in line with a number of clinical trials demonstrating that pseudoatrophy is a temporary phenomenon (i.e. first 3-6 months of treatment), characterized by accelerated reduction of edema, leading to nontissue-related brain volume loss as a response to anti-inflammatory therapies. ${ }^{24,25}$ In the second year of treatment, it has been convincingly demonstrated that disease-modifying treatments do slow brain atrophy progression. ${ }^{24-29}$ Over 36 months, patients who initiated GA40 treatment earlier showed a trend toward less GM volume loss than patients in the DS group $(-2.01 \%$ vs $-2.33 \%$, respectively; $p=0.07)$, especially between Months 12 and $36(-1.16 \%$ vs $-1.53 \%$, respectively; $p=0.015)$. In line with this finding, patients who initiated GA40 treatment earlier showed a trend toward less whole-brain volume loss than patients in the DS group, albeit not significantly, except for a trend between Months 12 and $36(-1.13 \%$ vs $-1.27 \%$, 
respectively; $p=0.08)$. These findings are in agreement with the results from a previous study that showed that GA reduced the rate of brain atrophy compared with placebo from Months 9 to $18(-0.6 \%$ vs $-1.0 \%$, respectively; $p=0.015$ ) and from Months 0 to 18 $(-1.5 \%$ vs $-2.0 \%$, respectively; $p=0.037)$ but not in the period between Months 0 and $9(-0.8 \%$ vs $-0.9 \%$, respectively; $p=0.37$ ) using the SIENA method. ${ }^{30}$ The differences in the number of patients analyzed for each of the brain volumetry MRI endpoints may account for the discernible treatment effect of early GA40 on GM and whole-brain atrophy. In addition, it has been shown that the early brain volume loss was specific to WM, not GM, as reported in a study of 45 MS patients on natalizumab therapy followed over 2 years, supporting a water shift effect related to decrease in inflammation. ${ }^{31}$ Finally, since changes in the volumes of thalamic and deep GM structures showed no differences between groups, it is possible that GA40 exerted a greater effect on cortical GM.

GA40 was well tolerated, with a safety profile similar to that of GA20, and no new safety signals were observed. Furthermore, no new safety signals in laboratory values, ECG, or vital signs were reported. As expected from prior clinical evaluation of GA40 safety, $3,11,32$ the incidence of injection-site reactions, including injection-site atrophy, was low for all patients exposed to GA40 during the OL phase, demonstrating the value of this regimen in limiting side effects, along with less frequent dosing.

In summary, treatment with GA40 conferred clinical benefit over 3 years of the GALA study, resulting in sustained low ARR and MRI lesion activity. Results from this OL extension of the GALA study further support the safe and effective use of GA40 in the RRMS patient population.

\section{Dedication}

This report is dedicated to the memory of Professor Omar Khan.

\section{Acknowledgments}

The authors thank the patients and site personnel involved with this study; all country principal investigators, the Data and Safety Monitoring Board, and the clinical steering committee; Svetlana Rubinchick (Teva Pharmaceuticals) for assistance with statistical analyses; and Peter Feldman, $\mathrm{PhD}$ (Teva Pharmaceuticals), and Rhonda Charles, PhD (Chameleon Communications International with funding from Teva Pharmaceutical Industries Ltd), for editorial assistance in the preparation of this report. This study was funded by Teva Pharmaceutical
Industries Ltd, Petach Tikva, Israel. A.B., O.K., P.R., K.S., and R.Z. were members of the steering committee and were site investigators. All authors reviewed the study report and contributed to its preparation. Trial Registration: NCT01067521 at www.ClinicalTrials.gov.

\section{Declaration of Conflicting Interests}

The author(s) declared the following potential conflicts of interest with respect to the research, authorship, and/ or publication of this article: O.K. has received compensation for consulting from Biogen Idec, Genzyme, and Novartis and for serving on speaker bureaus from Teva Pharmaceutical Industries Ltd, Novartis, and Biogen, and he has received research support from the NIH, NINDS, NMSS, Teva Pharmaceutical Industries Ltd, Biogen, Genzyme, Roche, and Novartis. P.R. has received compensation for speaking from Bayer, Biogen Idec, Boehringer Ingelheim, Novartis, Merck Serono, Teva Pharmaceutical Industries Ltd, and Genzyme and has served on steering committees for clinical trials for Novartis, Merck Serono, and Teva Pharmaceutical Industries Ltd. A.B. is a member of advisory boards and has served on steering committees for clinical trials for Bayer Schering, Merck Serono, Teva, Novartis, Biogen, Nycomed, Genzyme, and other companies. K.S. has received honoraria for advisory board membership, consulting, and speaking from Biogen Idec, Genzyme, ONO Pharma, Novartis, Bayer, Hoffmann La-Roche, Merck Serono, and Synthon. N.A., M.D.D., and S.K. are employees of Teva Pharmaceutical Industries Ltd. R.Z. has received compensation for speaking and consulting from Teva Pharmaceutical Industries Ltd, EMD Serono, Inc., Novartis, Claret, IMS Health, and Sanofi-Genzyme, and he has received research support from Biogen Idec, Teva Pharmaceutical Industries Ltd, Claret, InteKrin Coherus, IMS Health, Sanofi-Genzyme, Novartis, and EMD Serono, Inc.

\section{Funding}

The author(s) disclosed receipt of the following financial support for the research, authorship, and/or publication of this article: This study was supported by Teva Pharmaceutical Industries Ltd.

\section{GALA study group}

Principal investigators: O. Khan and P. Rieckmann. Study steering committee: A. Boyko, O. Khan, P. Rieckmann, K. Selmaj, and R. Zivadinov. Independent data monitoring committee: G. Cutter, R. Gold, and J. Wolinsky. MRI analysis center: R. Zivadinov, Ellen Carl, D.P. Ramasamy, M.G. Dwyer, N. Bergsland, J. Durfee, P. Polak, and Buffalo Neuroimaging Analysis Center, Department of Neurology, University of Buffalo, Buffalo, NY, USA. 


\section{Study sites}

Bulgaria: G. Angov, P. Bozhinov, M. Danovska, M. Daskalov, N. Deleva, K. Genov, L. Haralanov, S. Kastrev, K. Kmetska-Shotekova, I. Manchev, D. Maslarov, I. Milanov, E. Nedyalkov, I. Petrov, P. Shotekov, I. Staikov, E. Titianova, E. Vacheva, and Z. Zahariev. Croatia: S. Basic, M. Bosnjak Pasic, M. Habek, S. Soldo-Butkovic, and A. Vladic. Czech Republic: P. Hradilek, P. Kanovsky, and M. Vachova. Estonia: K. Gross-Paju, I. Kalbe, and G. Zjablov. Georgia: R. Shakarishvili and A. Tsiskaridze. Germany: P. Flachenecker, P. Friedemann, F. Heidenreich, P. Oschmann, G. Reifschneider, A. Simonow, B. Tackenberg, H. Tumani, R. Wenzel, and T. Ziemssen. Hungary: B. Clemens, I. Deme, P. Imre, K. Matyas, and M. Satori. Israel: J. Chapman. Italy: L. Grimaldi, T. Koudriavtseva, M. Rovaris, and F. Salvi. Lithuania: R. Kizlaitiene, D. Rastenyte, and S. Sceponaviciute. Poland: W. Brola, W. Fryze, E. Jasinska, A. Kaminska, J. Kapustecki, M. Kleczkowska, W. Kozubski, J. Krupa-Olchawa, E. Motta, R. Nowak, R. Podemski, A. Potemkowski, K. Selmaj, M. Sobkowiak-Osinska, M. StrzeleckaGorzynska, A. Tutaj, J. Zagorska, and J. Zbrojkiewicz. Romania: O. Bajenaru, R. Balasa, G. Boeru, A. Bulboaca, A. Campeanu, N. Carciumaru, A. Hancu, I. Marginean, M. Pereanu, W. Pop, C. Popescu, M. Simu, and C. Zaharia. Russia: V. Alifirova, A. Boyko, A. Fedyanin, A. Gustov, R. Magzhanov, N. Malkova, N. Maslova, S. Odinak, I. Poverennova, S. Perfilyev, S. Prokopenko, T. Romazina, A. Shutov, A. Sokoromets, N. Spirin, I. Stolyarov, and L. Volkova. South Africa: M. Isaacs, D. Lurie, C. Retief, and Z. Sacoor. Ukraine: N. Buchakchyyska, G. Chmyr, S. Kareta, T. Kobys, G. Kushnir, K. Loganovskyi, O. Moroz, S. Moskovko, T. Nehrych, V. Pashkovskyy, I. Pasyura, V. Pinchuk, V. Smolanka, O. Statinova, and A. Voloshchuk. United Kingdom: D. Rog and B. Sharrack. United States: G. Anderson, R. Aung-Din, M. Baker, A. Bass, J. Burch, H. Crayton, S. Delgado, B. Dihenia, G. Eubank, R. Fallis, W. Felton, J. Florin, G. Garmany, W. Grainger, C. Huffman, A. Jacobs, O. Khan, A. Mazhari, A. Minagar, A. Miravalle, R. Murray, D. Negroski, R.B. Neiman, G. Pardo, S.J. Shafer, C. Sheppard, D. Silver, V. Simnad, B. Steingo, D. Thoen, and D. Wynn.

\section{References}

1. Caporro M, Disanto G, Gobbi C, et al. Two decades of subcutaneous glatiramer acetate injection: Current role of the standard dose, and new high-dose lowfrequency glatiramer acetate in relapsing-remitting multiple sclerosis treatment. Patient Prefer Adherence 2014; 8: 1123-1134.
2. Boster A, Bartoszek MP, O'Connell C, et al. Efficacy, safety, and cost-effectiveness of glatiramer acetate in the treatment of relapsing-remitting multiple sclerosis. Ther Adv Neurol Disord 2011; 4: 319-332.

3. Copaxone (Prescribing information). Teva Neuroscience, Inc., Overland Park, KS, January 2014.

4. Data on file. Copaxone exposure. Teva Pharmaceutical Industries Ltd, Netanya, 30 June 2014.

5. Devonshire V, Lapierre Y, Macdonell R, et al. The Global Adherence Project (GAP): A multicenter observational study on adherence to disease-modifying therapies in patients with relapsing-remitting multiple sclerosis. Eur J Neurol 2011; 18: 69-77.

6. Verdun di Cantogno E, Russell S and Snow T. Understanding and meeting injection device needs in multiple sclerosis: A survey of patient attitudes and practices. Patient Prefer Adherence 2011; 5: 173-180.

7. Treadaway K, Cutter G, Salter A, et al. Factors that influence adherence with disease-modifying therapy in MS. $J$ Neurol 2009; 256: 568-576.

8. Al-Sabbagh A, Bennet R, Kozma C, et al. Medication gaps in disease modifying therapy for multiple sclerosis are associated with an increased risk of relapse: Findings from a national managed care database. J Neurol 2008; 255: S79.

9. Tan H, Cai Q, Agarwal S, et al. Impact of adherence to disease-modifying therapies on clinical and economic outcomes among patients with multiple sclerosis. Adv Ther 2011; 28: 51-61.

10. Remington G, Rodriguez $\mathrm{Y}$, Logan D, et al. Facilitating medication adherence in patients with multiple sclerosis. Int J MS Care 2013; 15: 36-45.

11. Khan O, Rieckmann P, Boyko A, et al. Three times weekly glatiramer acetate in relapsing-remitting multiple sclerosis. Ann Neurol 2013; 73: 705-713.

12. Comi G, Cohen JA, Arnold DL, et al. Phase III dosecomparison study of glatiramer acetate for multiple sclerosis. Ann Neurol 2011; 69: 75-82.

13. Zivadinov R, Havrdova E, Bergsland N, et al. Thalamic atrophy is associated with development of clinically definite multiple sclerosis. Radiology 2013; 268: 831-841.

14. Jenkinson $\mathrm{M}$, Bannister $\mathrm{P}$, Brady $\mathrm{M}$, et al. Improved optimization for the robust and accurate linear registration and motion correction of brain images. Neuroimage 2002; 17: 825-841.

15. Zivadinov R, Heininen-Brown M, Schirda CV, et al. Abnormal subcortical deep-gray matter susceptibilityweighted imaging filtered phase measurements in patients with multiple sclerosis: A case-control study. Neuroimage 2012; 59: 331-339. 
16. Smith SM, Zhang Y, Jenkinson M, et al. Accurate, robust, and automated longitudinal and crosssectional brain change analysis. Neuroimage 2002; 17: 479-489.

17. Smith SM, De Stefano N, Jenkinson M, et al. Normalized accurate measurement of longitudinal brain change. J Comput Assist Tomogr 2001; 25: 466-475.

18. Dwyer MG, Bergsland $\mathrm{N}$ and Zivadinov R. Improved longitudinal gray and white matter atrophy assessment via application of a 4-dimensional hidden Markov random field model. Neuroimage 2014; 90 : 207-217.

19. Patenaude B, Smith SM, Kennedy DN, et al. A Bayesian model of shape and appearance for subcortical brain segmentation. Neuroimage 2011; 56: 907-922.

20. Uher T, Horakova D, Bergsland N, et al. MRI correlates of disability progression in patients with CIS over 48 months. Neuroimage Clin 2014; 6 : 312-319.

21. Johnson KP, Brooks BR, Cohen JA, et al. Copolymer 1 reduces relapse rate and improves disability in relapsing-remitting multiple sclerosis: Results of a phase III multicenter, double-blind placebo-controlled trial. The Copolymer 1 Multiple Sclerosis Study Group. Neurology 1995; 45: 1268-1276.

22. Comi G, Filippi M and Wolinsky JS. European/ Canadian multicenter, double-blind, randomized, placebo-controlled study of the effects of glatiramer acetate on magnetic resonance imaging-Measured disease activity and burden in patients with relapsing multiple sclerosis. European/Canadian Glatiramer Acetate Study Group. Ann Neurol 2001; 49: 290-297.

23. Healy BC, Engler D, Glanz B, et al. Assessment of definitions of sustained disease progression in relapsing-remitting multiple sclerosis. Mult Scler Int 2013; 2013: 189624.
24. Zivadinov R, Reder AT, Filippi M, et al. Mechanisms of action of disease-modifying agents and brain volume changes in multiple sclerosis. Neurology 2008; 71: 136-144.

25. De Stefano N, Airas L, Grigoriadis N, et al. Clinical relevance of brain volume measures in multiple sclerosis. CNS Drugs 2014; 28: 147-156.

26. Vollmer T, Signorovitch J, Huynh L, et al. The natural history of brain volume loss among patients with multiple sclerosis: A systematic literature review and meta-analysis. J Neurol Sci 2015; 357: 8-18.

27. Tsivgoulis G, Katsanos AH, Grigoriadis N, et al. The effect of disease modifying therapies on brain atrophy in patients with relapsing-remitting multiple sclerosis: A systematic review and meta-analysis. PLoS ONE 2015; 10: e0116511.

28. Tsivgoulis G, Katsanos AH, Grigoriadis N, et al. The effect of disease-modifying therapies on brain atrophy in patients with clinically isolated syndrome: A systematic review and meta-analysis. Ther $A d v$ Neurol Disord 2015; 8: 193-202.

29. Branger P, Parienti JJ, Sormani MP, et al. The effect of disease-modifying drugs on brain atrophy in relapsing-remitting multiple sclerosis: A metaanalysis. PLoS ONE 2016; 11: e0149685.

30. Sormani MP, Rovaris M, Valsasina P, et al. Measurement error of two different techniques for brain atrophy assessment in multiple sclerosis. Neurology 2004; 62: 1432-1434.

31. Vidal-Jordana A, Sastre-Garriga J, Perez-Miralles F, et al. Early brain pseudoatrophy while on natalizumab therapy is due to white matter volume changes. Mult Scler 2013; 19: 1175-1181.

32. Wolinsky JS, Borresen TE, Dietrich DW, et al. GLACIER: An open-label, randomized, multicenter study to assess the safety and tolerability of glatiramer acetate $40 \mathrm{mg}$ three-times weekly versus $20 \mathrm{mg}$ daily in patients with relapsing-remitting multiple sclerosis. Mult Scler Relat Disord 2015; 4: 370-376.
Visit SAGE journals online journals.sagepub.com/ home/msj

@SAGEjournals 